\title{
Multiplexed bead-based immunoassay of four serum biomarkers for diagnosis of ovarian cancer
}

\author{
YONG-WAN KIM $^{1}$, SU MI BAE ${ }^{1}$, IN-WOOK KIM ${ }^{1}$, HAI-BO LIU ${ }^{1}$, HYO JOO BANG $^{1}$, \\ PANKAJ KUMAR CHATURVEDI ${ }^{1}$, GANTUMUR BATTOGTOKH ${ }^{1}$, HYUNSUN LIM $^{2}$ and WOONG SHICK AHN ${ }^{3}$ \\ ${ }^{1}$ Catholic Research Institutes of Medical Science, College of Medicine, The Catholic University of Korea, \\ ${ }^{2}$ Gangnam Severance Hospital Biomedical Research Center, Yonsei University College of Medicine, ${ }^{3}$ Department of \\ Obstetrics and Gynecology, College of Medicine, The Catholic University of Korea, Seoul, Republic of Korea
}

Received January 27, 2012; Accepted April 2, 2012

DOI: $10.3892 /$ or.2012.1829

\begin{abstract}
The purpose of the present study was to evaluate multiplex liquid assay-based measurement of multiple ovarian cancer-associated biomarkers such as hemoglobin, haptoglobin and apolipoprotein E, together with CA125, which has been widely used in the diagnosis of ovarian cancer, in order to provide a higher diagnostic power. We measured the serum levels of CA125, hemoglobin, haptoglobin and apolipoprotein $\mathrm{E}$ from the serum of 76 healthy individuals and 69 ovarian cancer patients using a multiplex liquid assay system, Luminex 100. The results were analyzed according to normal versus ovarian cancer, tumor stages and tumor histology. In addition, to validate the use of these biomarkers for the diagnosis of ovarian cancer, the sensitivity and specificity of each biomarker was analyzed by its receiver operating characteristics (ROC) curve. The serum levels of all four biomarkers in ovarian cancer patients were significantly higher than those of healthy individuals. When CA125 was combined with the biomarkers, the overall sensitivity and specificity were significantly improved in the ROC curve, which showed 95 and $75 \%$ sensitivity and specificity, respectively. At $95 \%$ specificity for all stages the sensitivity increased to $75 \%$ compared to $41 \%$ for CA125 alone. For stage I+II increased the sensitivity to $68 \%$ from $36 \%$ for CA125 alone. For stage III+IV the corresponding values were 100 and $95 \%$, respectively. Taken together, the new combination of hemoglobin, haptoglobin and apolipoprotein E with CA125 significantly improved both the sensitivity and the specificity of ovarian cancer diagnosis compared with those of individual biomarkers. These findings suggest the benefit of the combination of these markers for the diagnosis of ovarian cancer.
\end{abstract}

Correspondence to: Dr Woong Shick Ahn, Department of Obstetrics and Gynecology, College of Medicine, The Catholic University of Korea, 505 Banpo-dong, Seocho-gu, Seoul 137-040, Republic of Korea

E-mail: ahnlab1@catholic.ac.kr

Key words: CA125, apolipoprotein E, hemoglobin, haptoglobin, luminex, ovarian cancer

\section{Introduction}

Ovarian cancer has a very high mortality rate, since it tends to be asymptomic, which results in the vast majority of patients with ovarian cancer being diagnosed in advanced stages (stage III/IV) (1,2). The 5-year survival rate of patients with early stage cancer ranges from $50-95 \%$, but it is $<25 \%$ for those with advanced stage disease $(3,4)$. Given our knowledge about the steep decrease in survival rates relative to the stage at which the disease is diagnosed, it is reasonable to suggest that early detection remains the most promising approach with which to improve the long-term survival of ovarian cancer patients. Therefore, considerable efforts have been focused on the identification of diagnostic biomarkers for early detection of ovarian cancer $(5,6)$.

CA125 has been used as a serum marker of ovarian cancer for monitoring responses to chemotherapy, detecting disease recurrence, distinguishing malignant from benign pelvic masses, and potentially improving the designs of clinical trials. However, CA125 has proven to be a poor diagnostic tumor biomarker for early stage ovarian cancer (7). It is elevated above reference levels in only $50 \%$ of clinically detectable early stage disease, and is not infrequently elevated in patients with benign ovarian tumors $(8,9)$. In addition, CA125 levels are falsely elevated in pregnant women and women with detectable intraperitoneal pathologies that may alter the clearance of the antigen (10-12). Therefore, attempts have been made to combine or replace CA125 with other markers, and investigators have evaluated the ability of some established markers to improve the identification and prognosis of ovarian cancer $(8,13,14)$, thus indicating that the addition of one or several markers to CA125 would improve diagnostic and prognostic performance if sensitivity were improved without a loss in specificity. However, because the measurement of serum concentration of each putative biomarker with individual ELISAs requires considerable time, cost, and sample volumes in order to assess the combined effects of several markers, new methods or technologies for multiplexing must be developed.

The Luminex 100 bead-based system is a recently developed technology that provides multiplexing in a solution phase, resulting in it being particularly flexible and nondestructive for protein analysis. Each set of up to 100 uniquely color-coded 
Table I. Concentration of serum markers with clinicopathological findings in ovarian cancer patients.

\begin{tabular}{|c|c|c|c|c|c|}
\hline Characteristics & $\mathrm{N}(\%)$ & $\begin{array}{l}\text { CA125 } \\
\text { (U/ml) }\end{array}$ & $\begin{array}{l}\text { Hemoglobin } \\
(\mu \mathrm{g} / \mathrm{ml})\end{array}$ & $\begin{array}{l}\text { Haptoglobin } \\
\quad(\mu \mathrm{g} / \mathrm{ml})\end{array}$ & $\begin{array}{l}\text { Apolipo E } \\
\text { (ng/ml) }\end{array}$ \\
\hline Healthy normal (control) & $76(100 \%)$ & $11.5^{\mathrm{a}}$ & 69.0 & 347.6 & 100.1 \\
\hline Ovarian cancer patients & $69(100 \%)$ & & & & \\
\hline Age (years), mean (range) & $50.1 \pm 14.0(17-82)$ & & & & \\
\hline \multicolumn{6}{|l|}{ FIGO stage } \\
\hline I & $29(42.0 \%)$ & 42.3 & 188.8 & 479.0 & 157.0 \\
\hline II & $9(13.0 \%)$ & 230.9 & 992.7 & 1209.5 & 838.6 \\
\hline III & $19(27.5 \%)$ & 909.4 & 212.8 & 996.5 & 523.4 \\
\hline IV & $9(11.6 \%)$ & 943.7 & 995.6 & 1263.9 & 1318.5 \\
\hline \multicolumn{6}{|l|}{ Histological subtype } \\
\hline Serous & $33(47.8 \%)$ & 681.7 & 417.8 & 916.9 & 541.3 \\
\hline Mucinous & $10(14.5 \%)$ & 42.4 & 269.8 & 506.6 & 255.6 \\
\hline Clear cell & $9(13.0 \%)$ & 129.6 & 305.8 & 498.0 & 276.7 \\
\hline Endometrioid & $8(11.6 \%)$ & 58.9 & 402.7 & 648.6 & 312.9 \\
\hline Granulosa cell & $4(5.8 \%)$ & 13.6 & 147.8 & 478.2 & 91.7 \\
\hline Other & $5(7.2 \%)$ & 718.6 & 1167.3 & 1684.4 & 1334.0 \\
\hline
\end{tabular}

${ }^{\mathrm{a}}$ Values are presented as the mean.

polystyrene microspheres can be anchored with a different capture antibody. The use of detection antibodies labeled with biotin and streptavidin-R-phycoerythrin allows quantification of antigen-antibody reactions that occur on the microsphere surface through the measurement of the relative fluorescence intensity. Therefore, the system is capable of measuring up to 100 analytes simultaneously in a small sample volume $(<50 \mu \mathrm{l})$.

In this study, we measured four serum biomarkers of ovarian cancer, CA125, hemoglobin, haptoglobin, and apolipoprotein E, using a multimarker bead-based immunoassay system, and evaluated the combined effect of the four biomarkers for the diagnosis of ovarian cancer compared with those of the individual markers alone.

\section{Materials and methods}

Patients and samples. All patients were enrolled at St. Mary's Hospital of Catholic Medical School during the period from January 2001 to July 2007, according to the procedures approved by the Institutional Review Board of The Catholic University of Korea. This study was based on analyses of serum collected from patients with ovarian cancer $(n=69)$ and normal healthy females $(n=76)$. Patient serum was harvested before surgery or chemotherapy, and was then incubated for $30 \mathrm{~min}$ at room temperature, followed by centrifugation at $3,000 \mathrm{rpm}$ for separation. The serum was stored at $-70^{\circ} \mathrm{C}$ until they were used in experiments; frequent freezing and thawing were avoided. The stages and grades of tumors from the ovarian cancer patients were assigned according to the guidelines provided by the International Federation of Gynecology and Obstetrics (FIGO), and the enrolled groups were then divided according to age.
Conjugation of primary antibodies with microspheres. Four different kinds of microspheres $\left(1 \times 10^{6}\right.$ microspheres for each antibody, Biosource, Camarillo, CA) were prepared in each tube, and were then resuspended well by vortexing and sonication, followed by centrifugation for $2 \mathrm{~min}$ at $8,000 \mathrm{rpm}$. Supernatants were discarded, and the pellets were saved and washed once with $100 \mu \mathrm{l}$ saline. Monobasic sodium phosphate (80 $\mu 1$ of $100 \mathrm{mM})(\mathrm{pH}$ 6.2, Sigma-Aldrich, St. Louis, MO), $10 \mu \mathrm{l}$ of $50 \mathrm{mM}$ Sulfo-NHS (Pierce Biotechnology, Rockford, IL) and $10 \mu 1$ of $50 \mathrm{mM}$ EDC (Pierce Biotechnology) were added, and the solution was then incubated for $20 \mathrm{~min}$ at room temperature. After centrifugation $(8,000 \mathrm{rpm}, 2 \mathrm{~min})$, the pellets were saved and washed twice with $250 \mu 1$ of $50 \mathrm{mM}$ MES (pH 5.0, Sigma-Aldrich). After the removal of the supernatant, $500 \mu \mathrm{l}$ of MES was added to each tube including different microspheres. Following the addition of $0.5 \mu \mathrm{g}$ of each antibody [anti-CA125 (Fitzgerald Industries International, Inc., Concord, MA), anti-hemoglobin (Abcam, Cambridge, MA), anti-haptoglobin (Abcam), anti-apolipoprotein E (Fizgerald Industries International, Inc.)] in each tube, the tubes were incubated for $2 \mathrm{~h}$ on a shaker, which was protected from light. After the incubation, antibody-bound microspheres were pelleted by centrifugation for $2 \mathrm{~min}$ at $8,000 \mathrm{rpm}$, and $500 \mu \mathrm{l}$ of $1 \% \mathrm{BSA}$ buffer was then added. After additional incubation for $30 \mathrm{~min}$ at room temperature, the microspheres were washed twice with $1 \%$ BSA buffer and then stored at $4^{\circ} \mathrm{C}$ under protection from light.

Labeling biotins on the secondary antibodies. For labeling biotins on the secondary antibodies, a biotin labeling kit (Alpha Diagnostics International Inc., San Antonio, TX) was used according to the manufacturer's protocol. Briefly, biotin was 


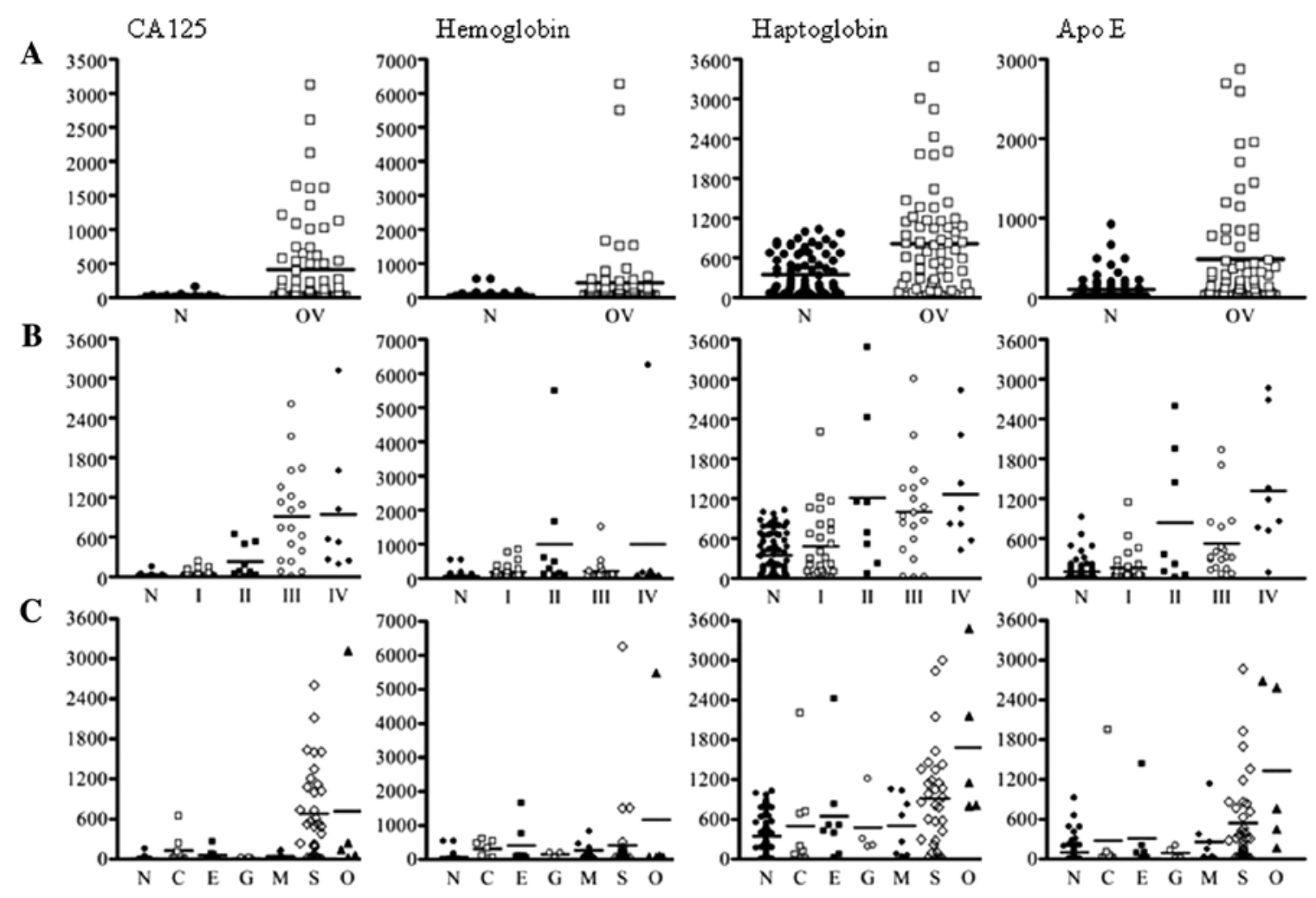

Figure 1. Scatter plots of concentrations of CA125, hemoglobin, haptoglobin, and apolipoprotein E. (A) Normal controls and ovarian cancer patients; (B) tumor stages in ovarian cancer patients; (C) different histological subtypes in normal controls and ovarian cancer patients. N, normal controls; C, clear cell; E, endometrioid; G, granulosa cell; $\mathrm{M}$, mucinous; $\mathrm{S}$, serous; $\mathrm{O}$, other.

added at a ratio of 1:10 (biotin:antibody). After incubation for $1 \mathrm{~h}$ at room temperature under protection from light, dialysis was performed with phosphate-buffered saline (PBS).

Analysis of samples by multiplex liquid array system, Luminex 100. The serum from healthy normal control and ovarian cancer patients were diluted to $1: 100$ in a buffer including 1\% BSA (Sigma-Aldrich) and 0.05\% Tween-20 (Sigma-Adrich). Fifty $\mu \mathrm{l}$ of each diluted serum were plated on a $1.2-\mu \mathrm{m}$ filter plate (96-well), to which 2,500 of each antibody-bound microsphere were added in $50 \mu$ l. After incubation for $2 \mathrm{~h}$ at room temperature under protection from light, they were washed twice with PBS buffer including $0.05 \%$ Tween-20. Streptavidin-R-phycoerythrin (100 $\mu$ l of $0.4 \mu \mathrm{g}$ ) (Sigma-Aldrich) was added to each well, and plates were then incubated for $30 \mathrm{~min}$, followed by two washes with PBS containing $0.05 \%$ Tween-20. The identification of antibody-bound microspheres and the screening of antigenantibody-bound microspheres were carried out by using Luminex 100 (Luminex Corp., Houston, TX) according to the manufacturer's protocol. Ranges of the concentrations of each antigen for standard curves were $10-250 \mathrm{U} / \mathrm{ml}$ for CA125, $1-1000 \mu \mathrm{g} / \mathrm{ml}$ for hemoglobin, $0.1-100 \mu \mathrm{g} / \mathrm{ml}$ for haptoglobin, and $0.5-50 \mathrm{ng} / \mathrm{ml}$ for apolipoprotein E. The data were analyzed by the BeadView program (Upstate, Charlottesville, VA).

Statistical analysis. The analysis of variance (ANOVA) test was used to assess the statistical significance of differences between the normal individuals and ovarian cancer patients. SigmaPlot (v12.0, Systat, Chicago, IL) and SAS (v9.1, SAS Institute, Cary, $\mathrm{NC)}$ was used for statistical analysis to determine the sensitivity, specificity, and the receiver operator characteristic (ROC) curve.

\section{Results}

Serum levels of ovarian tumor markers in normal control and ovarian cancer groups. The characteristics of patients and serum levels of ovarian cancer markers are shown in Table I. Concentration of serum biomarkers such as CA125, hemoglobin, haptoglobin, and apolipoprotein $\mathrm{E}$ in serum from healthy normal control and ovarian cancer patients was simultaneously measured by a multiplex liquid array system using microbeads coated with capture antibodies and biotin-labeled antibodies against each of the tumor markers and streptavidinR-phycoerythrin. The serum levels of all four tumor markers were significantly higher in ovarian cancer patients than that in normal controls (Fig. 1A). First, we compared the serum levels of these four tumor markers according to the tumor stages (Fig. 1B). The serum levels of CA125 were gradually elevated with tumor stage. Also, the other three tumor markers were significantly increased in ovarian cancers compared with that in normal controls. Next, we attempted to compare the serum levels of four tumor markers according to histologic types of ovarian cancer (Fig. 1C). The serum levels of CA125, haptoglobin, and apolipoprotein $\mathrm{E}$ were the highest in serous type compared with those in the other types, as the case numbers 

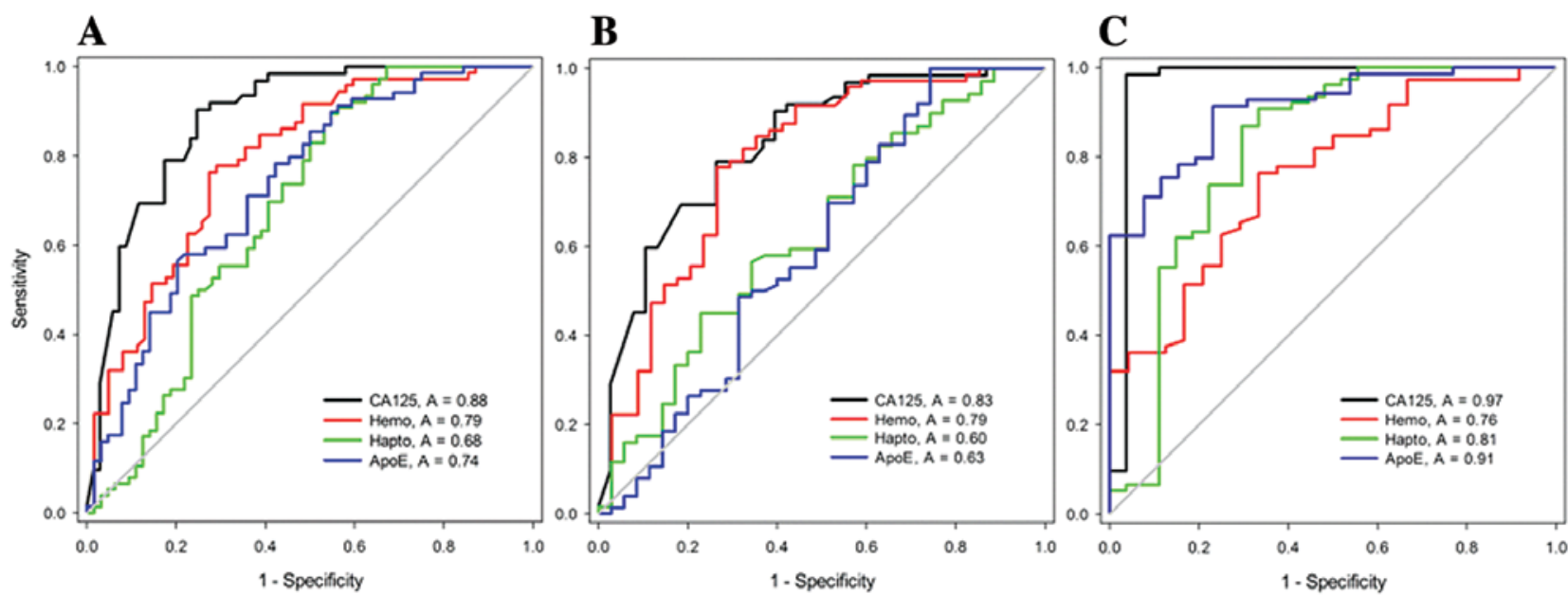

Figure 2. ROC (receiver operator characteristic) discriminating ovarian cancer from normal controls. The curves shown were obtained by processing quantified raw data by SigmaPlot 12.0 version software and the sensitivity/specificity values were predicted from the area under the curves and the calculated data. ROC curves for CA125, hemoglobin, haptoglobin, and apolipoprotein E alone: (A) healthy controls versus ovarian cancer; (B) healthy controls versus stages I to II ovarian cancer; (C) healthy controls versus stages III to IV ovarian cancer.

Table II. Sensitivities and specificities.

\begin{tabular}{|c|c|c|c|c|c|c|c|c|c|c|}
\hline \multirow[b]{2}{*}{ Patient group } & \multicolumn{2}{|c|}{ CA125 } & \multicolumn{2}{|c|}{ Hemoglobin } & \multicolumn{2}{|c|}{ Haptoglobin } & \multicolumn{2}{|c|}{ Apolipo E } & \multicolumn{2}{|c|}{ All markers } \\
\hline & $\mathrm{SN}^{\mathrm{a}}$ & $\mathrm{SP}^{\mathrm{b}}$ & SN & SP & SN & SP & SN & SP & SN & SP \\
\hline \multirow[t]{5}{*}{ All stages } & 99 & 42 & 99 & 13 & 99 & 32 & 99 & 16 & 99 & 24 \\
\hline & 95 & 62 & 95 & 42 & 95 & 36 & 95 & 27 & 95 & 70 \\
\hline & 90 & 75 & 90 & 52 & 90 & 43 & 90 & 44 & 90 & 87 \\
\hline & 59 & 90 & 36 & 90 & 7 & 90 & 7 & 90 & 86 & 90 \\
\hline & 41 & 95 & 32 & 95 & 6 & 95 & 16 & 95 & 75 & 95 \\
\hline \multirow[t]{5}{*}{ Stage I+II } & 99 & 13 & 99 & 15 & 99 & 26 & 99 & 11 & 99 & 29 \\
\hline & 95 & 45 & 95 & 44 & 95 & 26 & 95 & 14 & 95 & 29 \\
\hline & 90 & 61 & 90 & 56 & 90 & 29 & 90 & 23 & 90 & 71 \\
\hline & 45 & 90 & 32 & 90 & 8 & 90 & 17 & 90 & 76 & 90 \\
\hline & 36 & 95 & 22 & 95 & 4 & 95 & 16 & 95 & 68 & 95 \\
\hline \multirow[t]{5}{*}{ Stage III+IV } & 99 & 89 & 99 & 8 & 99 & 44 & 99 & 23 & 99 & 97 \\
\hline & 95 & 96 & 95 & 33 & 95 & 52 & 95 & 46 & 95 & 97 \\
\hline & 90 & 96 & 90 & 38 & 90 & 67 & 90 & 77 & 90 & 100 \\
\hline & & & 36 & 90 & 7 & 90 & 71 & 90 & 100 & 90 \\
\hline & & & 36 & 95 & 7 & 95 & 62 & 95 & 100 & 95 \\
\hline
\end{tabular}

${ }^{\mathrm{a} S e n s i t i v i t y ;}{ }^{\mathrm{b}}$ Specificity.

for patients with clear cell and granulosa cell histology were too small.

Comparison of the sensitivity and specificity between four tumor markers alone and the combination of four markers for the diagnosis of ovarian cancer. We compared the sensitivity and specificity between each marker alone and the four markers in combination in order to diagnose ovarian cancer using receiver operating characteristic (ROC) analysis. In this study, we used cut-off values of $35 \mathrm{U} / \mathrm{ml}, 71.6 \mu \mathrm{g} / \mathrm{ml}$,
$1,007 \mu \mathrm{g} / \mathrm{ml}$, and $248.4 \mathrm{ng} / \mathrm{ml}$ for CA125, hemoglobin, haptoglobin, and apolipoprotein $\mathrm{E}$, respectively, for better diagnostic accuracy for the samples tested. By using these cut-off values, we were able to minimize the rates of falsepositive and false-negative findings in the differentiation of normal controls from subjects with ovarian cancer. The sensitivity and specificity of individual markers with CA125, hemoglobin, haptoglobin, and apolipoprotein E were 88.5 and $75.3 \%, 80.0$ and $66.1 \%, 68.0$ and $59.4 \%$, and 73.6 and $59.4 \%$, respectively (Fig. 2A). 

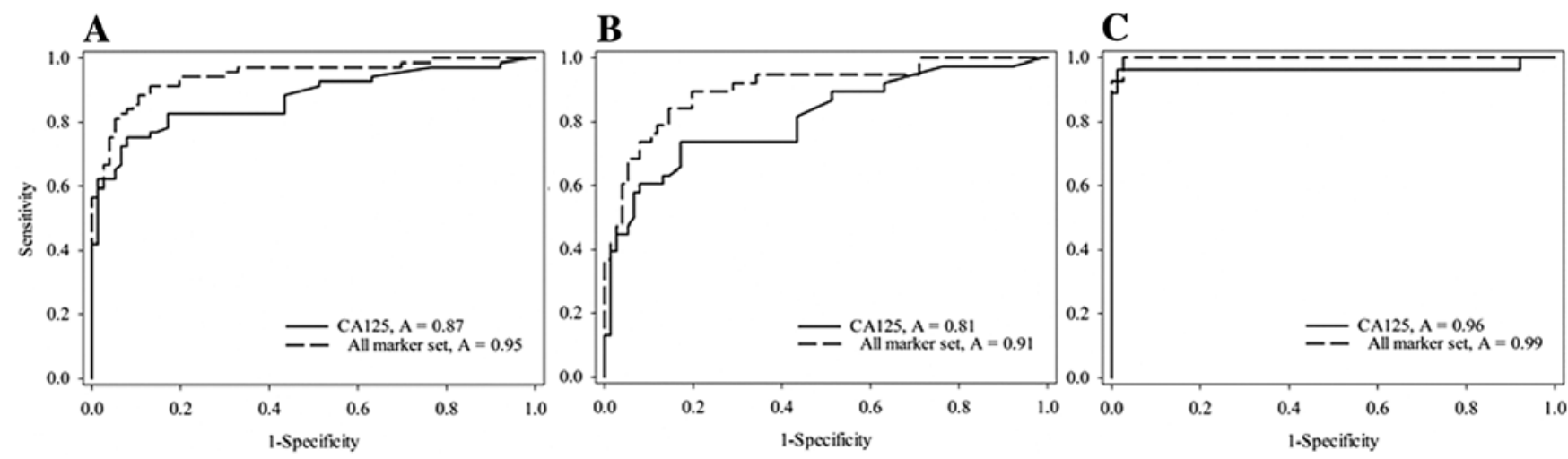

Figure 3. ROC discriminating ovarian cancer from normal controls using the four-biomarker set. The curves shown were obtained by processing quantified raw data by SAS 9.1 version software and the sensitivity/specificity values were predicted from the area under the curves and the calculated data. ROC curves for CA125 and the four-biomarker set: (A) healthy controls versus ovarian cancer. The overall difference in AUCs between four biomarkers and CA125 was statistically significant $(\mathrm{P}<0.011)$; (B) healthy controls versus stages I to II ovarian cancer $(\mathrm{P}<0.028)$; (C) healthy controls versus stages III to IV ovarian cancer $(\mathrm{P}<0.33)$

The sensitivity and specificity of the individual markers for early-stage (stages I and II) were 82.9 and $63.2 \%, 78.9$ and $70.2 \%, 62.8$ and $48.6 \%$, and 59.6 and $57.1 \%$, respectively (Fig. 2B). And the sensitivity and specificity of the individual markers for late-stage (stages III and IV) were 96.5 and $96.3 \%, 75.9$ and $66.7 \%, 81.2$ and $70.4 \%$, and 90.8 and $76.9 \%$, respectively (Fig. 2C). The sensitivities and specificities for discriminating between ovarian cancer and healthy tissue are shown in Table II. For CA125 alone, at 90\% (95\% CI, 82-96\%) specificity, overall sensitivity was 59\% (95\% CI, 46-72\%), $45 \%$ (95\% CI, 32-58\%) for stages I+II, and 98\% (95\% CI, 91-99\%) for stages III+IV. At 95\% (95\% CI, 82-96\%) specificity, overall sensitivity was $41 \%$ (95\% CI, 32-58\%) for all stages, 36\% (95\% CI, 24-48\%) for stages I+II and 96\% (95\% CI, 88-99\%) for stages III+IV.

When CA125 was combined with the biomarkers (hemoglobin, haptoglobin, and apolipoprotein E), the overall sensitivity and specificity were significantly improved in the ROC curve, which showed 95 and $75 \%$ sensitivity and specificity, respectively (Fig. 3A). At a very high sensitivity of $99 \%$ there was a loss in specificity to $24 \%$ for all four tumor markers compared to $42 \%$ for CA125 alone. At this sensitivity, however, the specificity was higher for all markers in the stage I+II patient group (Fig. 3B). The strength of the markers at this high sensitivity is restricted to the stage III+IV group with a specificity at $97 \%$ (Fig. 3C). At $95 \%$ specificity for all stages the sensitivity increased to $75 \%$ compared to $41 \%$ for CA125 alone. For stage I+II increased the sensitivity to $68 \%$ from $36 \%$ for CA125 alone. For stage III+IV the corresponding values were, respectively, 100 and $95 \%$, suggesting that the four biomarker set classified early-stage cancers with $68 \%$ sensitivity and late-stage cancers with $100 \%$ sensitivity at $95 \%$ specificity, which was significantly higher than CA125 alone.

\section{Discussion}

In the present study, we evaluated, for the first time, a new combination of four known biomarkers of ovarian cancer, CA125, hemoglobin, haptoglobin, and apolipoprotein E, in an attempt to improve the sensitivity and specificity of the diagnosis of ovarian cancer. Moreover, this study effectively presented the validation of the use of a multiplex liquid assay system for the simultaneous detection of several biomarkers for the diagnosis of ovarian cancer.

CA125 has been a potentially useful marker for diagnosis and prognosis after treatment (surgery or conventional therapies) of ovarian cancer, but it is often not elevated in clinically-detected ovarian cancers, and is also frequently elevated in women with benign ovarian cancers (15-17). The cut-off $35 \mathrm{U} / \mathrm{ml}$ for CA125 we used is generally accepted (18). Due to the vulnerable points of CA125 as a biomarker of ovarian cancer (19), combining one or more other tumor markers with CA125 might improve the sensitivity and specificity of the diagnosis of ovarian cancers or the earlier detection of such cancers.

We have identified and verified hemoglobin- $\alpha$ and $-\beta$ as new serum biomarkers of ovarian cancer using proteomic technologies and ELISA (20). Following our identification of hemoglobin as an ovarian cancer biomarker, four serum proteins, including hemoglobin $\beta$, was identified as serum biomarkers of early stage ovarian cancer using micro-LC-MS/ MS and ELISA analysis (21). Consistent with these previous reports, the serum level of hemoglobin in ovarian cancer was significantly higher than that in normal controls in this study. The level of hemoglobin was higher in stage II cancers, but was not stage-dependent, while that of CA125 was much higher in late stages (III and IV) of ovarian cancer than in early stages. These findings suggest that combination of CA125 with hemoglobin could compensate for the weakness of CA125 in the detection of early stage ovarian cancer. However, to evaluate the validation of hemoglobin as a biomarker for early detection, extended case numbers of early and late stage cancers are required for comparison, as the numbers of cases presented here are small.

In a manner similar to other acute-phase proteins, haptoglobin also originates mainly from the liver, and elevation of this peptide could be observed in infections, inflammation, and various malignant diseases, including lung and bladder cancers (22-24), leukemia (25), breast cancer (26), and urogenital tumors (27). Haptoglobin- $\alpha$ has been suggested as 
a serum biomarker using surface-enhanced laser desorption and ionization, and subsequently identified this as the $\alpha$ chain of haptoglobin using ELISA (28). In addition, it was reported that significantly elevated haptoglobin concentrations were associated with poor survival rates in women with ovarian cancer, and chemotherapeutic treatment reduced the serum level of haptoglobin, thus indicating a role of haptoglobin in the monitoring of patients undergoing chemotherapy (29), as well as in the diagnosis of ovarian cancer $(30,31)$.

Apolipoprotein E, was among the genes that were highly up-regulated in ovarian cancer, as identified by serial analysis of gene expression (SAGE); this was further validated through immunohistochemical analysis (32). In addition, the expression of apolipoprotein E was frequently detected in ovarian serous carcinomas, the most common and lethal type of ovarian cancer, but not in serous borderline tumors or normal ovarian surface epithelium $(33,34)$. Even though these findings strongly suggested that apolipoprotein E might function as a potent biomarker of ovarian cancer, the present study is the first trial to use apolipoprotein $\mathrm{E}$ as a serum biomarker of ovarian cancer. Our data show that the serum level of apolipoprotein E was elevated in ovarian cancer patients, and was the highest in serous carcinoma. These data were in agreement with the previously reported result that apolipoprotein $\mathrm{E}$ was highly expressed in ovarian serous carcinoma (35). Thus, these combined results indicate that apolipoprotein $\mathrm{E}$ in serum might be a product that is released from tumors, and could be a direct predictor of ovarian cancer.

However, when applied individually, three of the markers studied here did not surpass CA125 in their sensitivities and specificities in the diagnosis of ovarian cancer. Combining individual markers has been attempted by other researchers as one strategy to enhance the overall ovarian cancer detection rate (19,36-38). We applied the combination of the three serum markers with CA125, and compared the sensitivities and specificities between the combination of the four markers and each marker alone. Results from ROC curve analysis show that combining four biomarkers had a much improved sensitivity over that of each biomarker alone. At a high sensitivity of $90 \%$, combining four biomarkers resulted in a slight increase in the specificity to $87 \%$ compared to $75 \%$ for CA125 alone. At a high specificity of $90 \%$, there was a gain in sensitivity to $27 \%$ for the four biomarker set. At a $95 \%$ specificity for all stages the sensitivity increased to $75 \%$ compared to $41 \%$ for CA125 alone. The four biomarker set classified early-stage cancers with $68 \%$ sensitivity and late-stage cancers with $100 \%$ sensitivity at $95 \%$ specificity. The sensitivity and specificity of this panel for stage III+IV are comparable to results with a four biomarker panel selected from 96 candidate antigens measured by immunoassays with multiplex techniques (39). The high specificity and corresponding increases in sensitivity for all four biomarkers have merit in ovarian cancer screening trials. Thus, a large number of ovarian cancer patients and a healthy control cohort would be required to further improve the specificity and sensitivity of the combined biomarkers in both retrospective and prospective clinical trials and lead to increased survival $(6,40)$.

We performed a multimarker bead-based immunoassay for the detection of these biomarkers in the serum from normal control and ovarian cancer patients using a multiplex liquid assay system, Luminex 100. This immunoassay system has several benefits for the immunoassay using clinical samples compared with the conventional enzyme-linked immunosorbent assay techniques and proteomic based analyses (41). i) This system requires only a small sample volume for simultaneous detections for several markers, so in the event that several assays for the detection of several markers should be required with very small or rare samples from patients (39), this system is very beneficial. ii) Time and cost can be saved due to multiplexing. iii) When new biomarkers are identified from high-throughput screening and need to be verified, they can be measured with previously established markers at the same time and in the same well, resulting in reducing experimental errors or variations. However, there are some difficulties inherent to the set up for multiplexing. Since proper concentrations of antibodies in bead-antibody conjugation according to the antibodies present, the concentration of each antibody should be experimentally determined. A good pair of capture antibody and detection antibody should be determined, and cross-reactivity among different antibodies for multiplexing should be avoided. Several commercially available Luminex multiplex panels were compared with conventional commercial ELISAs for measurement of biomarkers in human plasma that are associated with obesity and inflammation (42). The correlation between Luminex multiplexed assays and ELISAs was good for some analytes, but some with very low plasma concentrations showed low assay sensitivity and poor correlations, thus suggesting that the Luminex multiplex system might be considered when attempting to detect analytes present at very low concentrations in serum. Although the Luminex multiplex assay system has the complexities mentioned above, this technology will be very useful and convenient in clinical studies with a large number of samples once the most appropriate conditions are determined.

The present study showed significant improvement of sensitivity for the diagnosis of ovarian cancer when using a combination of new serum biomarkers, including CA125, hemoglobin, haptoglobin, and apolipoprotein E, using a multiplex liquid assay system. Further studies are going to be extended to a large number of ovarian cancer patients in early and late stages, as well as healthy women, in order to confirm the validity of the combination of these markers for the diagnosis at an early stage of ovarian cancer.

\section{Acknowledgements}

The study was supported by the National Research Foundation of Korea (NRF), Seoul, Republic of Korea (Grant no. 5-2011A0154-00120).

\section{References}

1. Badgwell D and Bast RC Jr: Early detection of ovarian cancer. Dis Markers 23: 397-410, 2007.

2. Bast RC Jr: Early detection of ovarian cancer: new technologies in pursuit of a disease that is neither common nor rare. Trans Am Clin Climatol Assoc 115: 233-248, 2004.

3. Morice P, Brehier-Ollive D, Rey A, et al: Results of interval debulking surgery in advanced stage ovarian cancer: an exposednon-exposed study. Ann Oncol 14: 74-77, 2003.

4. Integrated genomic analyses of ovarian carcinoma. Nature 474: 609-615, 2011. 
5. Donach M, Yu Y, Artioli G, et al: Combined use of biomarkers for detection of ovarian cancer in high-risk women. Tumour Biol 31: 209-215, 2010.

6. Hensley ML: A step forward for two-step screening for ovarian cancer. J Clin Oncol 28: 2128-2130, 2010.

7. Liede A, Karlan BY, Baldwin RL, Platt LD, Kuperstein G and Narod SA: Cancer incidence in a population of Jewish women at risk of ovarian cancer. J Clin Oncol 20: 1570-1577, 2002.

8. Tcherkassova J, Abramovich C, Moro R, Chen C, Schmit R and Gerber A: Combination of CA125 and RECAF biomarkers for early detection of ovarian cancer. Tumour Biol 32: 831-838, 2011

9. Einhorn N, Sjovall K, Knapp RC, et al: Prospective evaluation of serum CA 125 levels for early detection of ovarian cancer. Obstet Gynecol 80: 14-18, 1992.

10. Aslam N, Ong C, Woelfer B, Nicolaides K and Jurkovic D: Serum CA125 at 11-14 weeks of gestation in women with morphologically normal ovaries. BJOG 107: 689-690, 2000.

11. Menon U and Jacobs IJ: Recent developments in ovarian cancer screening. Curr Opin Obstet Gynecol 12: 39-42, 2000.

12. Predanic M: Differentiating tubal abortion from viable ectopic pregnancy with serum CA-125 and beta-human chorionic gonadotropin determinations. Fertil Steril 73: 522-525, 2000

13. Zhang Z, Bast RC Jr, Yu Y, et al: Three biomarkers identified from serum proteomic analysis for the detection of early stage ovarian cancer. Cancer Res 64: 5882-5890, 2004.

14. Gupta D and Lis CG: Role of CA125 in predicting ovarian cancer survival - a review of the epidemiological literature. J Ovarian Res 2: 13, 2009.

15. Jacobs I and Bast RC Jr: The CA 125 tumour-associated antigen: a review of the literature. Hum Reprod 4: 1-12, 1989.

16. Kim KA, Park CM, Lee JH, et al: Benign ovarian tumors with solid and cystic components that mimic malignancy. AJR Am J Roentgenol 182: 1259-1265, 2004.

17. Helzlsouer KJ, Bush TL, Alberg AJ, Bass KM, Zacur H and Comstock GW: Prospective study of serum CA-125 levels as markers of ovarian cancer. JAMA 269: 1123-1126, 1993.

18. Bast RC Jr, Klug TL, St John E, et al: A radioimmunoassay using a monoclonal antibody to monitor the course of epithelial ovarian cancer. N Engl J Med 309: 883-887, 1983.

19. Clarke CH, Yip C, Badgwell D, et al: Proteomic biomarkers apolipoprotein $\mathrm{A} 1$, truncated transthyretin and connective tissue activating protein III enhance the sensitivity of CA125 for detecting early stage epithelial ovarian cancer. Gynecol Oncol 122: 548-553, 2011.

20. Woong-Shick A, Sung-Pil P, Su-Mi B, et al: Identification of hemoglobin-alpha and -beta subunits as potential serum biomarkers for the diagnosis and prognosis of ovarian cancer. Cancer Sci 96: 197-201, 2005.

21. Kozak KR, Su F, Whitelegge JP, Faull K, Reddy S and FariasEisner R: Characterization of serum biomarkers for detection of early stage ovarian cancer. Proteomics 5: 4589-4596, 2005.

22. Beckman G, Eklund A, Frohlander N and Stjernberg N: Haptoglobin groups and lung cancer. Hum Hered 36: 258-260, 1986.

23. Benkmann HG, Hanssen HP, Ovenbeck R and Goedde HW: Distribution of alpha-1-antitrypsin and haptoglobin phenotypes in bladder cancer patients. Hum Hered 37: 290-293, 1987.

24. Abdullah M, Schultz H, Kahler D, et al: Expression of the acute phase protein haptoglobin in human lung cancer and tumor-free lung tissues. Pathol Res Pract 205: 639-647, 2009.
25. Mitchell RJ, Carzino R and Janardhana V: Associations between the two serum proteins haptoglobin and transferrin and leukaemia. Hum Hered 38: 144-150, 1988.

26. Awadallah SM and Atoum MF: Haptoglobin polymorphism in breast cancer patients form Jordan. Clin Chim Acta 341: 17-21, 2004.

27. Dunzendorfer U, Jung K and Ohlenschlager G: Transferrin, C3 complement, haptoglobin, plasminogen and alpha 2-microglobulin in patients with urogenital tumors. Eur Urol 6: 232-236, 1980.

28. Ye B, Cramer DW, Skates SJ, et al: Haptoglobin-alpha subunit as potential serum biomarker in ovarian cancer: identification and characterization using proteomic profiling and mass spectrometry. Clin Cancer Res 9: 2904-2911, 2003.

29. Zhao C, Annamalai L, Guo C, et al: Circulating haptoglobin is an independent prognostic factor in the sera of patients with epithelial ovarian cancer. Neoplasia 9: 1-7, 2007.

30. Diamandis EP: Analysis of serum proteomic patterns for early cancer diagnosis: drawing attention to potential problems. J Natl Cancer Inst 96: 353-356, 2004

31. Ahmed N, Barker G, Oliva KT, et al: Proteomic-based identification of haptoglobin-1 precursor as a novel circulating biomarker of ovarian cancer. Br J Cancer 91: 129-140, 2004.

32. Hough CD, Sherman-Baust CA, Pizer ES, et al: Large-scale serial analysis of gene expression reveals genes differentially expressed in ovarian cancer. Cancer Res 60: 6281-6287, 2000.

33. Chen YC, Pohl G, Wang TL, et al: Apolipoprotein E is required for cell proliferation and survival in ovarian cancer. Cancer Res 65: 331-337, 2005

34. Shih Ie M and Kurman RJ: Ovarian tumorigenesis: a proposed model based on morphological and molecular genetic analysis. Am J Pathol 164: 1511-1518, 2004.

35. McIntosh MW, Drescher C, Karlan B, et al: Combining CA 125 and SMR serum markers for diagnosis and early detection of ovarian carcinoma. Gynecol Oncol 95: 9-15, 2004

36. Rosen DG, Wang L, Atkinson JN, et al: Potential markers that complement expression of CA125 in epithelial ovarian cancer. Gynecol Oncol 99: 267-277, 2005.

37. Anderson GL, McIntosh M, Wu L, et al: Assessing lead time of selected ovarian cancer biomarkers: a nested case-control study. J Natl Cancer Inst 102: 26-38, 2010.

38. Palmer C, Duan X, Hawley S, et al: Systematic evaluation of candidate blood markers for detecting ovarian cancer. PLoS One 3: e2633, 2008

39. Yurkovetsky Z, Skates S, Lomakin A, et al: Development of a multimarker assay for early detection of ovarian cancer. J Clin Oncol 28: 2159-2166, 2010.

40. Zhang B, Barekati Z, Kohler C, et al: Proteomics and biomarkers for ovarian cancer diagnosis. Ann Clin Lab Sci 40: 218-225, 2010.

41. West-Norager M, Kelstrup CD, Schou C, Hogdall EV, Hogdall CK and Heegaard NH: Unravelling in vitro variables of major importance for the outcome of mass spectrometry-based serum proteomics. J Chromatogr B Analyt Technol Biomed Life Sci 847: 30-37, 2007

42. Liu MY, Xydakis AM, Hoogeveen RC, et al: Multiplexed analysis of biomarkers related to obesity and the metabolic syndrome in human plasma, using the Luminex-100 system. Clin Chem 51: 1102-1109, 2005. 